\title{
KNOWLEDGE MANAGEMENT FOR PROCESS PLANNING
}

\author{
Ketil Bø
}

IDI, NTNU, No-7490 Trondheim, Norway.E-mail: Ketil.Bo@idi.ntnu.no

\begin{abstract}
Knowledge Management offers enterprises a considerable competitive edge. Together with manufacturing features extracted from CAD models, the knowledge can be used for augmenting several manufacturing processes such as automatic process planning. Xplano is a Feature Recognition and Knowledge Management system using Manufacturing features, company resources and a knowledge base of rules containing "best practice" in the enterprise to automatically generate process plans.
\end{abstract}

Key words: Knowledge management, Process planning, Mechanical industry, Feature recognition, Resource database, Knowledge database, ISO STEP, 3D Visualization, Rule based reasoning

\section{INTRODUCTION}

Knowledge management is capturing, organizing, and storing knowledge and experiences of individual workers and groups within the company and making this information available to others in the organization.

Mechanical industry has invested heavily in software for computer aided design (CAD) and computer aided manufacturing (CAM). Increasing functionality for $\mathrm{CAD} / \mathrm{CAM}$ is steadily reducing the amount of routine work connected to these activities.

On the other hand, the experts who decides WHERE and HOW to manufacture each product have limited access to modern tools.

To keep up with the designers and the manufacturing (more variants, shorter lifecycles etc.), the process planner is forced to look up his old plans from earlier work, and cut and paste from similar existing products. He is

Please use the following format when citing this chapter:

Bø, Ketil, 2006, in International Federation for Information Processing (IFIP), Volume 207, Knowledge Enterprise: Intelligent Strategies In Product Design, Manufacturing, and Management, eds. K. Wang, Kovacs G., Wozny M., Fang M., (Boston: Springer),

pp. 121-126. 
captured in routine work. His knowledge is not available for others, he has no time for developing new and more efficient solutions, and the knowledge will leave the company with him.

It is important for the competitiveness of the company to take care of "best practice" and make available the knowledge and the experience of the production process from those who know to those that need to know.

\section{PROCESS PLANNING}

The information associated with a machined mechanical part progresses through several steps, from its specification in a CAD system, through its interpretation in terms of manufacturing features, the associated sequence of machining operations to finally the tool paths for all operations. Each of these steps can be regarded as a transformation that produces a new representation of the object in another domain of discourse. Unlike most mathematical mappings, however, these transformations are interpretations of the object using knowledge about each successive domain to infer information that is not explicitly present in the model.

Process planning systems support the planning of the manufacturing procedure flow for a product. Here, both the manufacturing process and the manufacturing sequence must be determined. With basis in the geometry, the available resources and the company specific experience and knowledge, procedures for the production of work pieces are determined.

The planning of manufacturing sequences can be easier realized by using manufacturing features by means of which a tight coupling between geometry and manufacturing can be represented. For the interpretation of work piece data, a close connection between design and process planning must exist.

Recognition of manufacturing features, based on the CAD model, should be carried out as an integrated part of process planning to simplify the detection of tools and machines.

The process planning result mainly originate from the work piece, features, material, tools, machine hierarchies and the decision sequences for production planning. A high performance, object oriented form of representation is required, whereas the representation of decision sequences makes rule processing advantageous.

Databases should be employed for storage of resources and rules. The ability to modularize knowledge- and resource data bases is required for more complex planning systems in order to facilitate understanding of the program structure. 


\section{PRODUCTION KNOWLEDGE MANAGEMENT}

The production knowledge consist of the resources available, the "best practice" of where, how and in what sequence the features of a product are produced in the company.

Xplano is a software system designed to take over this routine work. At the same time it gives the enterprise a modern tool to accumulate experience and ensure continuous process improvements.

The software is a visualization and knowledge management system, making all the production knowledge in the enterprise, available for those who need to know. In addition it also has a reasoning engine that uses the knowledge in the system to decide the best methods, tools and process data for any product feature. This makes Xplano capable of generating a detailed process plan for the products according to the knowledge and experience accumulated from the local manufacturing experts.

For chip removing production, the resources are systematized in a Resource database with Machine tools, Fixtures, Rotating tools, Turning tools and Raw material. The Knowledge is systematized in a Knowledge database with Manufacturing methods, Machine tools rules, Fixture rules and Operation order rules.

Some large international companies have been successful with rule based systems that are able to decide the manufacturing process for a limited family of similar products. The problem with these systems are that a wider range of products gives a complexity in rules that will easily create conflicts in certain areas, and/or lack of exact rules in other areas. To cover other product families, another expert system with many of the same elements is required. Some of the same data and the same rules will be created and maintained for many different applications which will absorb a considerable amount of resources from system people and process designers.

Xplano's goal is to make a generic software with enterprise specific Resource- and Knowledge data bases and a flexible reasoning engine able to decide the manufacturing process for all the products in the enterprise, still being both easier to use and easier to maintain than the existing systems based on conventional programming and exact rules. This approach lowers the "break even" point for the investment, and the software will be profitable also for small and medium size enterprises.

Knowledge management (KM) is the management of the company's individual and collective knowledge. The ultimate benefits are overall improved efficiency and reduced time from design to production, as well as cost reduction through reduced time in process planning and optimal production. 
Promoting information sharing, motivating employees to stay with the firm, forging partnerships for knowledge acquisition is the benefit that makes industrial companies becoming increasingly aware of the need to manage individual and collective knowledge. The advantages deriving from knowledge management are not only explained by company size, specialization or research \& development efforts. Knowledge management also stimulates innovation, a productivity factor. Preserving a company's skills and expertise, acquiring and disseminating new knowledge, is important factors to improve the competitiveness.

Different factors are promoting knowledge management:

Companies have to manage a more complex world because of changing technologies.

They are concerned about the loss of skills caused by today's extreme mobility of personnel. For all these reasons, companies are striving to motivate their employees to stay with the firm, alternatively take care of the expert knowledge if the expert leaves the company.

\section{XPLANO}

Xplano get its "expert knowledge" from the Knowledge Management System (KMS) built and maintained by the production engineer himself in the individual enterprise.

The KMS includes decision rules for all the steps in the design of a manufacturing process together with a Resource database containing information about the manufacturing resources available. The decision rules are expert knowledge, agreed by the manufacturing engineers in the company as the best known practice, and linked up to the preferred resources and process data for all the different conditions the experts can think of.

Automatic Process Design starts with a "feature extraction" process from the geometry model written in the international standard ISO STEP format (most advanced CAD systems are capable of storing the design model in ISO STEP format).

Output from the" feature extraction" process is a feature file, written in XML format, with all the information available for the manufacturing process. If this information is not complete, it is possible to fill in additional requirements, before the file is processed by the Reasoning Engine.

The Visualization Module visualize both the geometry and the extracted $3 D$ feature model in a way optimized for Process planers, both for visual control and alternatively as a tool for manual or template based Process Planning. 
The Reasoning Engine reads the feature file, consults the KMS and puts together a detailed description of the best possible production process for the component, using the best fit resources available.

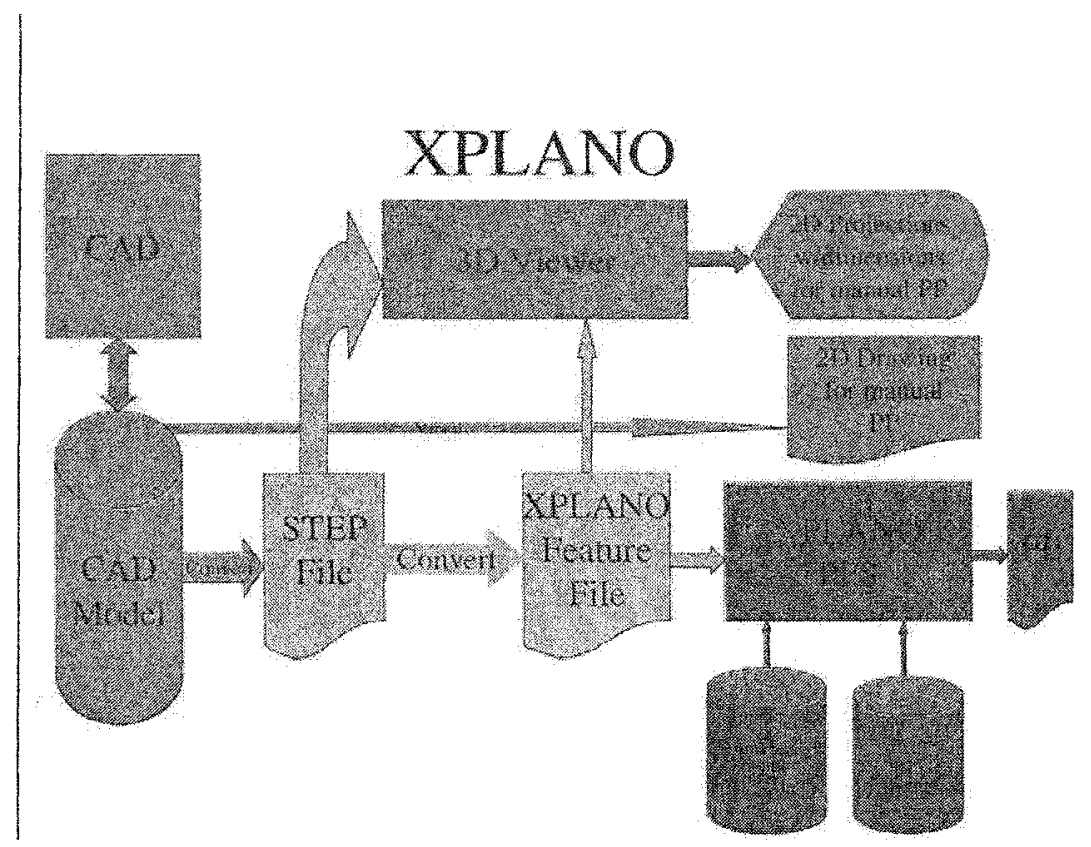

Figure 1. The integrated Xplano System

Artificial Intelligence techniques will assist the reasoning engine to take a decision when rules are in conflict or missing, and give the software the ability to learn from possible corrections made by the experts when the decision from the system is not acceptable.

The software is designed as a framework that must be filled in with the relevant rules and resources of the individual enterprise. The process specialist himself will enter the decision rules of the enterprise concerning methods, materials, machine tools, adapters etc..

The concept of Xplano is to collect as much product information as possible from the design file of the CAD system, and us it together with the decision rules from the company specific Knowledge database and the resources available from the Resource databases where the content are created and maintained by the local experts. 


\section{BENEFITS}

Significant benefits can result from the production planning system only. In a survey of twenty-two large and small companies using generative-type CAPP systems, the following estimated cost savings were achieved [4]:

$58 \%$ reduction in process planning effort.

$10 \%$ saving in direct labor.

$4 \%$ saving in material.

$10 \%$ saving in scrap.

$12 \%$ saving in tooling.

$6 \%$ reduction in work-in-process.

In addition, there are a number of other intangible benefits.

\section{CONCLUSION}

Manufacturing Knowledge management (MKM) is an efficient way to take care of company specific knowledge and experience. In addition the knowledge can be used to support and augment manufacturing processes such as generation of process plans.

Xplano is a Manufacturing Knowledge Management (MKM) system for mechanical industries that collect and systemize resources and process knowledge in an enterprise and makes it available for everybody involved.

In addition the system also extracts and visualizes manufacturing features from the CAD model and automatically generates a process plan containing "best practice" of the company.

\section{REFERENCES}

1. Han, J.H., Pratt, M. And Regli, W.C. (2000). Manufacturing Feature recognition from Solid Models: A Status Report. IEEE Transactions on Robotics and Automation. Vol 16, No 6.

2. $\mathrm{B} ø \mathrm{~K}$. (2006) Production Knowledge Management for the Mechanical Industry, $C A D$ User, Vol 18 No 12.

3. Vandenbrande, J.H. and Requicha, A.G. (1994) Geometric computation for recognition of spatial interacting machining features Advances in feature based manufacturing, North Holland: Elsevier,

4. Crow, K. A. Computer Aided Process Planning. DRM Associates

5. Unigraphics: "Hybrid Modeling Fundamentals" Electronic Data Systems Corporation, Version 13.0, Unigraphics Division

6. Krause, F.L. and Schlingheider, J. (1995) Development and Design With KnowledgeBased Software Tools - An Overview. Expert Systems with applications. Vol 8 No. 2 pp233-248. 